\title{
A Low Cost Method of Snow Detection on Solar Panels and Sending Alerts
}

\author{
Seyedali Meghdadi and Tariq Iqbal
}

\begin{abstract}
Photovoltaic systems are often installed in climates with considerable amount of snowfall and freezing rain in winter. It has been observed that the snow accumulation on a solar panel affects its performance and decreases the energy output. Snow on solar panels should be cleared as soon as possible to generate the maximum power. $A$ low cost method of snow detection on solar panels found on field tests is proposed in this paper. The designed system is based on a low cost open-source Arduino Uno microcontroller that measures voltage and current output of a solar panel, and output of a LDR representing the irradiance. Arduino is also connected to a WIFI network and can send messages over the internet. Based on the sensors data, an algorithm is designed to accurately detect snow on solar panels and notify the owner via twitter about the current status. The designed low cost and very low power system has been tested in St. John's, Newfoundland, Canada $\left(47^{\circ} 34^{\prime} 28.9^{\prime \prime} \mathrm{N} 52^{\circ} 44^{\prime} 07.8^{\prime \prime} \mathrm{W}\right)$ for three months of winter 2014. This paper presents details of the designed low cost alert system, algorithm and its performance results.
\end{abstract}

Index Terms - Snow detection, Arduino application, melting performance, solar energy, renewable energy.

\section{INTRODUCTION}

Solar panels are often installed in areas that receive some snow fall during winter months. In lower temperatures solar panels produced more output power due to reduced internal losses. Roughly $74 \%$ of PVs are installed in countries that experience some amount of snowfall [1], [2]. Energy reduction from a snow covered module can occur in three ways; a) diffusion of short wave through snow, b) Albedo reflection to the exposed rear of a module, and c) conduction from parts of PV not covered with snow. Although Panels are not physically damaged by the severe winter conditions, snow accumulation or ice could lead to decrease in energy output as long as panels are covered by precipitation. Published studies show that depending on orientation of PV modules and meteorological factors, the snow losses from a PV system can be as high as $20 \%$ for a low profile system to $0.3-2.7 \%$ for a highly exposed 28 degree roof mounted system [3], [4]. Therefore, solar panels should be cleared as soon as possible. Snow removal can be done in several ways. For instance, increasing tilt angle using a stepper motor not only would lead to slide the gathered snow, but also steeper tilt angles cause less snow to accumulate and therefore less power loss due to snowfall [2]. Furthermore, it was observed that snow shedding might occur due to sunlight or rise of temperature.

Manuscript received June 18, 2014; revised August 21, 2014.

The authors are with Memorial University of Newfoundland, Canada (email: s.meghdadi@mun.ca,tariq@mun.ca).
Snow shedding takes place in form of either melt on the modules (see Fig. 1(a)) or sheet sliding (see Fig. 1(b)) as some incident radiation would penetrate the layer of snow and melt the snow-module layer to produce a water layer leading to snow sliding.

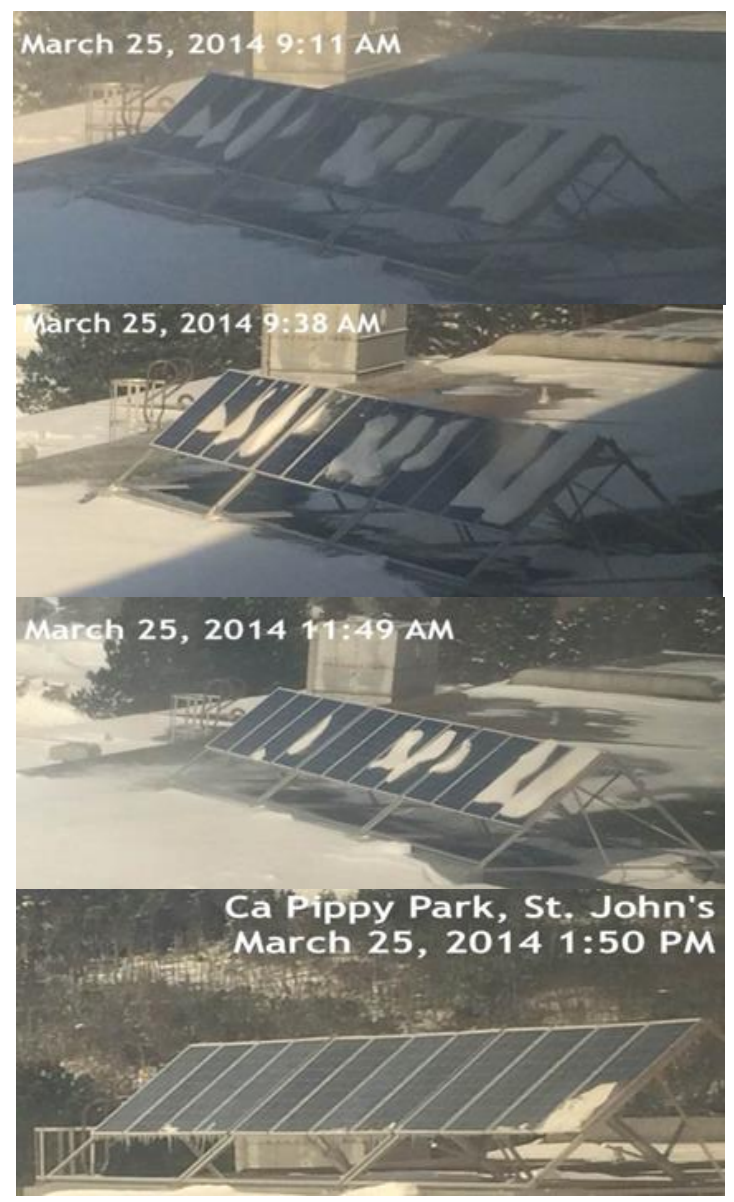

Fig. 1. (a) Snow melting.

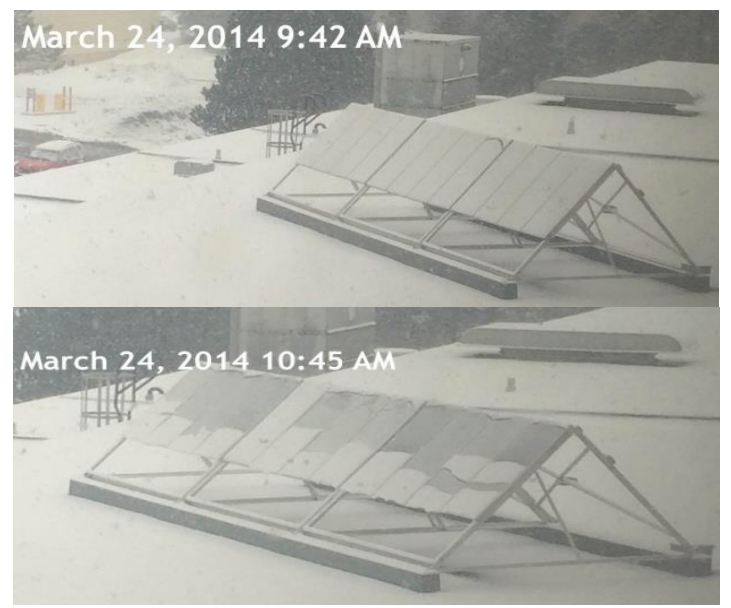

Fig. 1. (b) Sheet sliding. 


\section{PREVIOUS STUdIES AND WORKS}

There are some studies on effects of snowfall on PV systems.

In 1979 at Natural Bridges National Monument a simple linear empirical correlation to determine expected PV output was determined to find expected PV output. However $56 \%$ of the data were discarded in that study due to issues with pyrometer being obscured by snow or data logger reliability issues. A theoretical study on snow shedding was performed by Ross in 1995 with the goal of improving the battery charging performance in remote sites [5]. Recent studies have relied on continuous clearing of a set of control modules to have a reference or on comparison of actual system output to a modelled system output over a specific time period [6]. Utilizing satellite imaging to identify times when a photovoltaic plant is covered with snow was also studied in [7]. Aforementioned works and solutions are not only too expensive to be implemented but also not accurate. Moreover, some data logging systems are commercially available capable of detecting snow on solar panels by comparing outputs of two panels next to each other. Such detection systems consume significant power, not capable of sending alerts and are expensive. This paper presents a low cost system and a novel algorithm established on field test results to accurately detect snow on panels and notify the owner to clean the panels by means of twitter messages. No dedicated host PC or data logging system is required since it exploits a low cost and low power Aduino Uno board along with a wifi shield.

\section{SYSTEM OVERVIEW}

As shown in Fig. 2, the system is comprised of a solar panel along with its Maximum Power Point Tracker (MPPT) to obtain maximum power by adjusting the current drawn. Moreover, a current sensor, a voltage sensor and a Light Dependent Resistor (LDR) are used to measure irradiance and current status of PV panel. These three sensors provide enough data that enables the Arduino to determine if there is enough augmentation of snow on the panel.

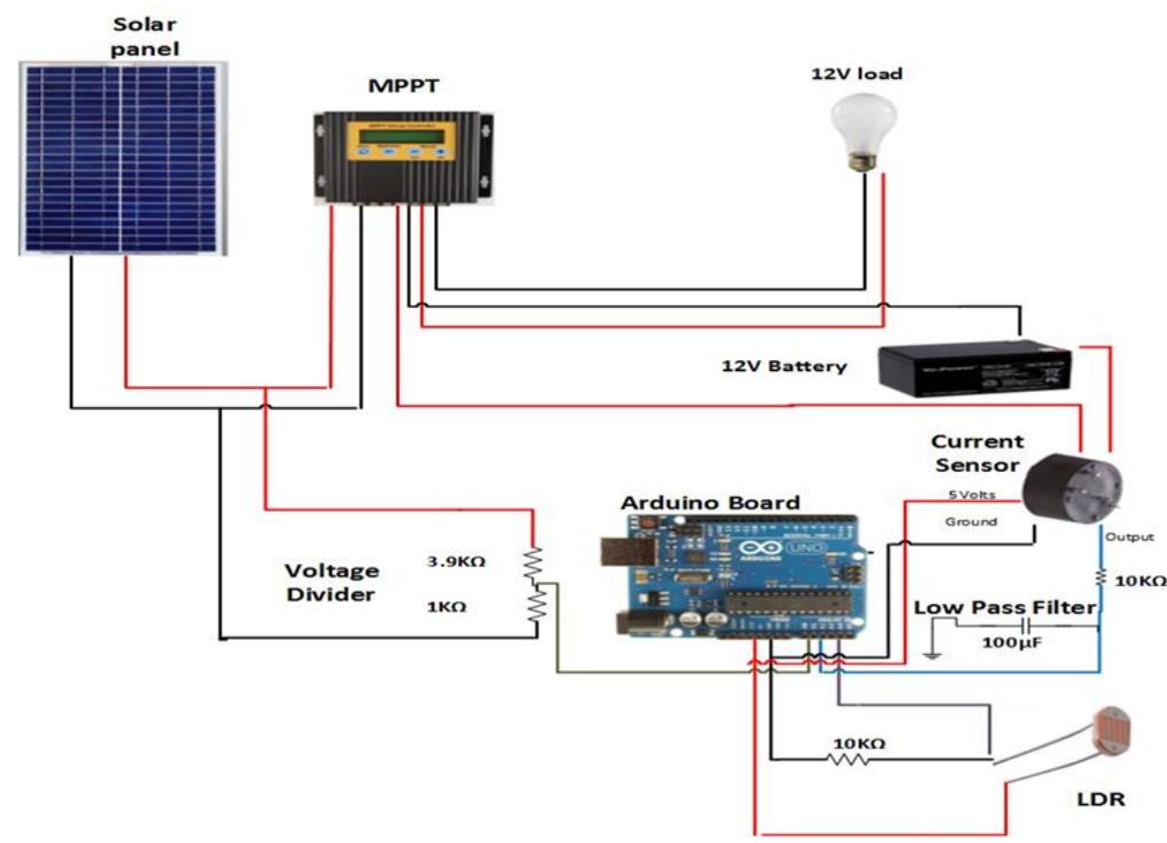

Fig. 2. System circuit diagram.

The Arduino Uno is a microcontroller board based on the ATmega328. It has 14 digital input/output pins (of which 6 can be used as PWM outputs), 6 analog inputs, a $16 \mathrm{MHz}$ ceramic resonator, a USB connection, a power jack, and a reset button. Its supply voltage limit is 6-20 $\mathrm{V}$ and it can be powered directly from a $12 \mathrm{~V}$ PV panel. Two voltage dividers are used to bring input voltages to microcontroller in measuring range.

One for connecting the panel output voltage to the analogue input pin of the Arduino and the other one for LDR (see Fig. 2). Also, output voltage of the current sensor is delivered to the board through a low pass filter in order to only read the low frequency responses. Sensors are connected to the board in company with its Wifi shield powered by the PV panel through adaptor input. Furthermore, a $12 \mathrm{~V}$ battery is connected to the MPPT output and a $12 \mathrm{~V}$ light bulb is used as a load connected to the same node.

\section{ALGoRITHM}

During four winter months of 2014, St. John's, Newfoundland got more than $4 \mathrm{~m}$ of snow. The designed system was closely observed and tested during these winter months. Data was collected in a variety of atmospheric conditions and types of snow collected on the panels. During the experiment it was noted that five centimeters of snow on panels could be a distinguishable feature affecting the PVs performance and is used as a check point in the algorithm.

Fig. 3 depicts the average reading of each sensor (in digital unit) within three months observation categorized based on atmospheric conditions. As the hour angle of a point on the Earth's surface is the angle through which the earth would turn to bring the meridian of the point directly under the sun, two hours' time interval from noon to $2 \mathrm{pm}$ is considered in this chart to exclude the effect of hour angle $(120 / 2=60$ data points for each day). 


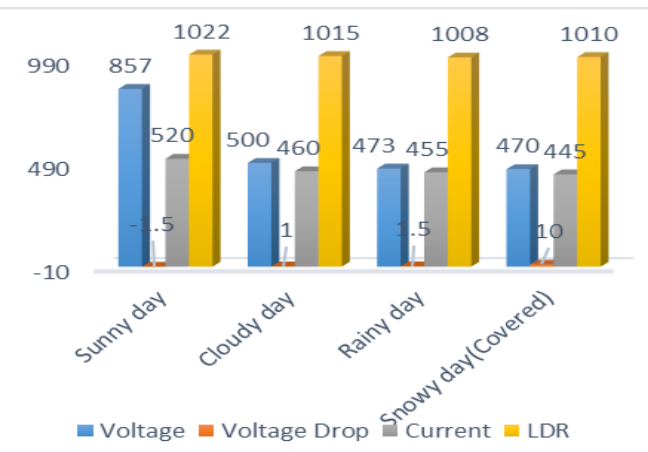

Fig. 3. Average of sensors readings during three months (12pm-2pm).

It can be seen that current and voltage readings in rainy and snowy days are quite similar. On the other hand, LDR reading which represents solar irradiance in snowy days is more than rainy days.

Although several similarities were found between a rainy day and a snowy day during the observations based on sensors readings, it was found that thick overcast of clouds can be categorized and recognized from snow by the value of the panel voltage drop i.e. the difference in the current voltage reading and the previous voltage reading. Seeking for the moments that enough snow is gathered, as demonstrated in Fig. 3, not only the algorithm checks all the sensors to be in certain scope, but also calculates the voltage drop which is a marker of snow detection.

Two promising scenarios are considered to keep the track of snow build up on the solar panels. First, as snow is accumulating on the panel, the algorithm starts computing the number of times that the criterion is reached. Second, present weather is rainy, cloudy or sunny and there exists snow whether from the day before or simply because panel wasn't cleared after the first tweet. Common property in both scenarios is voltage drop due to snow accumulation; however, in the first case there would be a smaller drop compared to the later.

Short time scale is not required as we are not interested in logging and saving many data points on a SD card. On the other hand, the aforementioned second scenario becomes complex as in a cloudy evening current sensor readings and voltage drop could have several similarities with a snowy weather. In order to solve the problem, time step was decreased and tuned to two minutes. This provided enough data to make decision and store on a small SD card. Based on three months of observation, two minutes time scale was determined most suitable to apprehend the ratio of voltage drop to detect snow build up.

The flowchart shown in the Fig. 4 essentially depicts the snow detection method. First step assigns previous voltage and counters including $i, j$ and $\mathrm{k}$ to zero. Here " $j$ " represents the first and " $k$ " represents the second scenario.

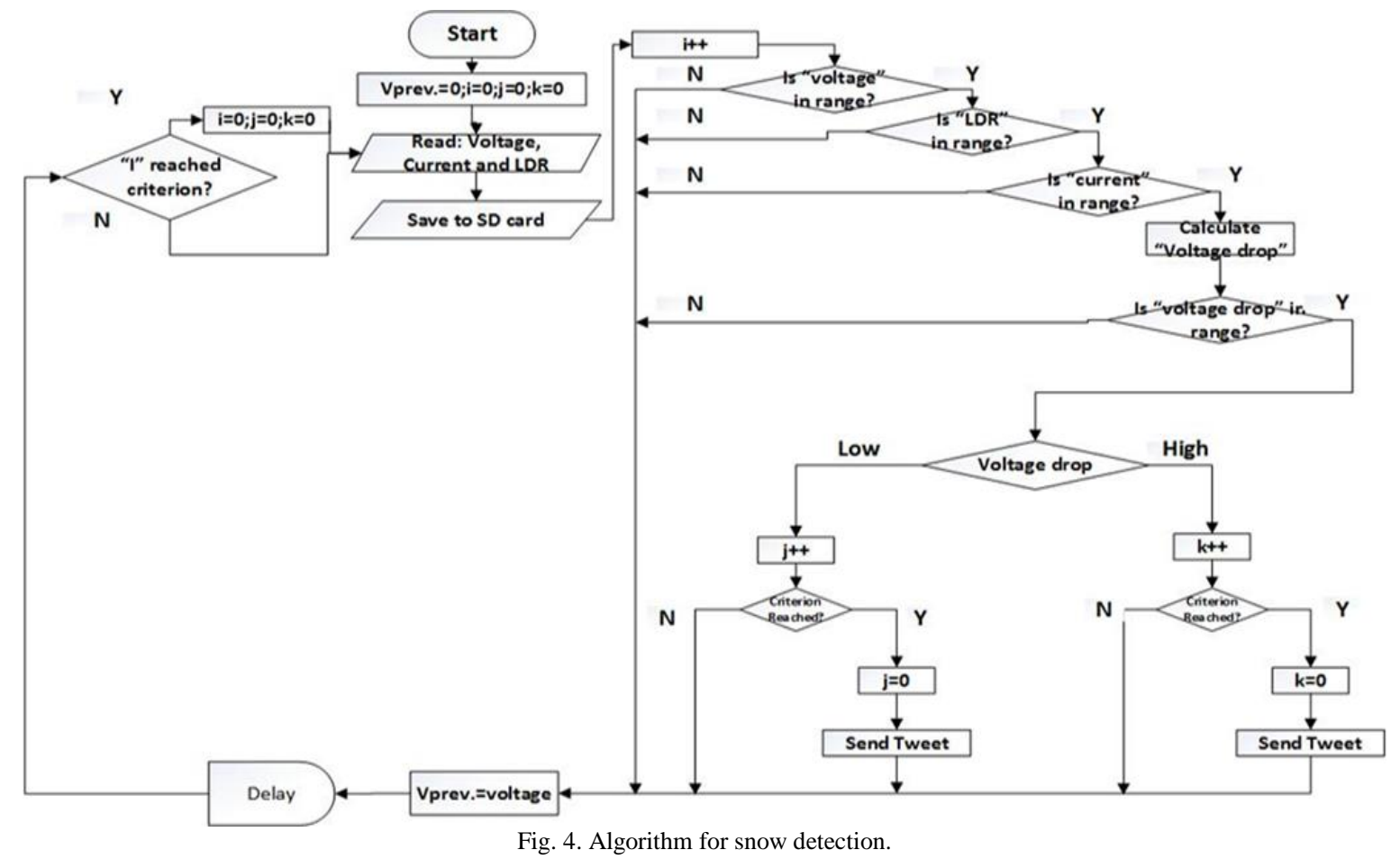

It sets $i, j$ and $k$ to zero in the end of the day. Subsequently, Arduino monitors voltage, current and LDR and record them on the SD-card and then total counter number increases by one. In the second step, it inspects voltage, LDR and current sensor to estimate the recorded numbers based on defined scope (defined range for voltage, current and LDR determined based on observations), which is necessary but not sufficient to indicate snow accumulation. Details may be found on code provided in the Appendix. When the calculated voltage drop is in the range it adds one to the number of times the criterion is reached (low stands for between three and ten and High for greater than ten defined in the criterions located after $i++$ and $j++$ in the above flowchart).

If " $j$ " or " $k$ " satisfies the condition (considered $j==6$ or $k==2$ ), the counter will be set equal to zero and sends tweet and both indicate snow build up equally. These threshold values were set, tuned and validated during the experiment. In the next step either after tweeting or when any of the conditions are not met, current voltage will be set equal to previous voltage along with a delay in order to calculate voltage drop. Finally, it assesses the number of times the whole process is accomplished and after one day it resets all the counters. If we approximately consider the time between 
sunrise and sunset equal to 15 hours, we would have $i=450$ since the criterion for " $i$ " is $15 \times 60 / 2$.

\section{ARDUINO SOFTWARE}

In the Arduino code after assigning the input and output pins and defining variables, connecting to the Internet is done. Arduino shield will not connect to networks using WPA2 Enterprise encryption so a WPA network was used [8]. The next step is logging to twitter. Twitter Library is a library for Arduino to tweet on Twitter via the Arduino Ethernet Shield [9]. In order to make the library work for wifi shield some modifications need to be done to the twitter.cpp and twitter.h files in library. The twitter library works by connecting arduino to a specific website which in turn will connect to the twitter server. This workaround is because the twitter authentication is a bit code heavy which will be difficult to implement on the arduino. Instead in this code heavy part is implemented on the website and arduino instructs the website to tweet [10].

Other than the main loop, two loops are defined as void loops. One for sending the tweets and the other for writing sensor values on a SD-card. Code also checks the criterions and calls the tweeter loop. Implemented method as described above in the Fig. 3 is tracking the inputs from the sensors and calculating voltage drop. Suppose the condition is met, it starts counting based on the two probable scenarios described above. After the counter reaches a specific number contingent upon snow mass, a tweet will be sent and thereupon both counters will be reset. Moreover, a delay of two minutes in the loop using delay commands in the program was found to be necessary and sufficient condition.

\section{EXPERIMENTAL RESULTS}

The designed system was tested during the winter 2014 at the Faculty of Engineering and Applied Science, Memorial University of Newfoundland. During this time a lot of data and time stamped pictures of panel's status was collected and the algorithm was fine tuned.

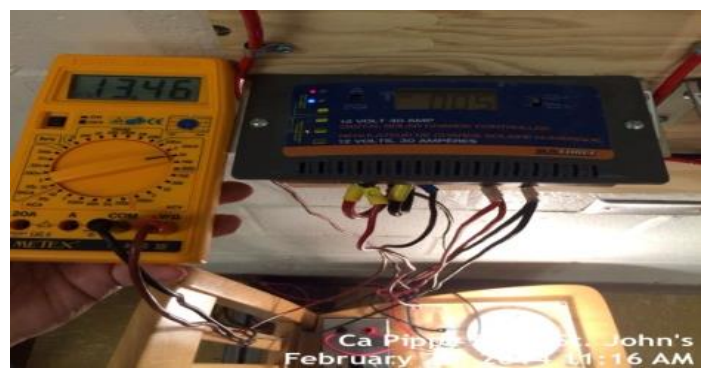

Fig. 5. System Setup.

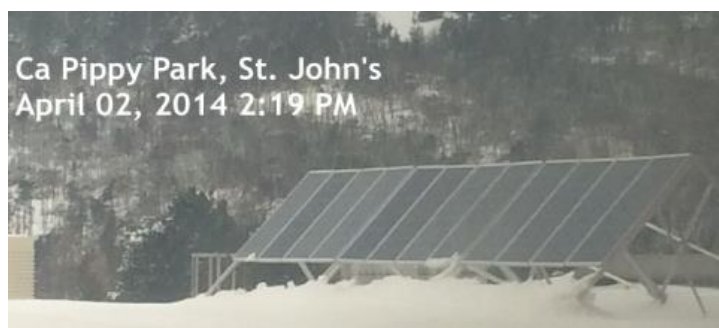

Fig. 6. Clean panels.

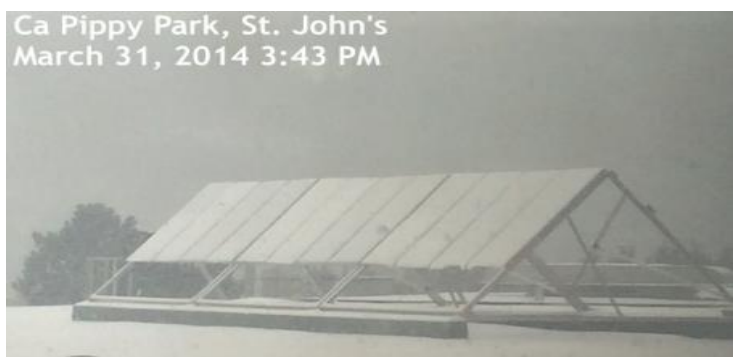

Fig. 7. Snow on panels.

Fig. 5 depicts the system setup in the lab indicating the MPPT value when the load is on. The multimeter showed the panels's output voltage which indicates a node voltage other than the parallel node of battery and load. All data points are also stored on a SD-card inserted on the Arduino Wifi shield (also available on author's twitter account). Fig. 6 shows a clear panel while in Fig. 7 accumulation of snow on the panels is displeyed pointing the various feasible working condition. Fig. 8, Fig. 9 exhibit tweeted messages to author's twitter account according to the state perceived in Fig. 6 provided with time and date stamp as a reference.

\section{CONCLUSION}

The designed system is capable of detecting more than five centimeters of snow accumulation as it significantly affected the PVs performance. The novel algorithm of snow detection and a low cost system as described above has been developed and validated. The paper presented details of system setup and program written for the Arduino. Such a low cost and low power alert system for PV panels is essential since proper assessment of snow related losses can help improve the system performance and maintenance leading to more energy in climates experiencing snowfall.

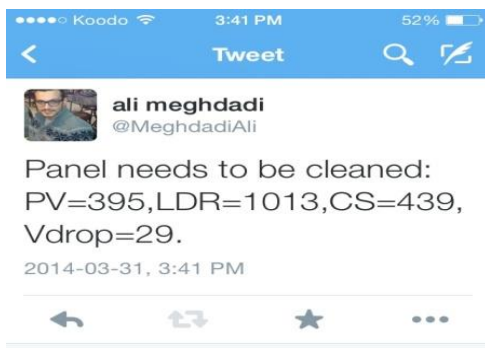

Fig. 8. Tweeted message.

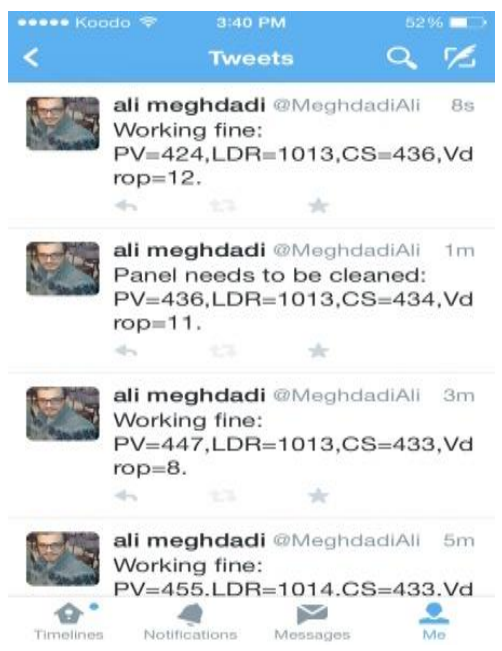

Fig. 9. Twitter messages. 


\section{FUTURE WORK}

It is required to investigate a mathematical formulation relating climate data to voltage and current sensors readings for solar panels. Moreover, methods to mitigate snowfall losses such as developing the idea of shedding the snow by means of increasing the slope of the module should be studied thoroughly as it could be the most economical manner of removing snow accumulation on solar panels. Finally, these approaches must be evaluated and taken into account for future system configuration.

\section{ACKNOWLEDGMENT}

This work was supported by the NSERC and Memorial University of Newfoundland.

\section{REFERENCES}

[1] B. Gerd, B. Schiebelsberger, W. Weber, C. Vodermayer, M. Zehner, and G. Kummerle, "An approach to the impact of snow on the yield of grid connected PV systems," in Proc. European PVSEC, 2006.

[2] L. Powers, J. Newmiller, and T. Townsend, "Measuring and modeling the effect of snow on photovoltaic system performance," in Proc. 35th IEEE Photovoltaic Specialists Conference, 2010, pp. 000973-000978.

[3] B. L. Brench, "Snow-covering effects on the power output of solar photovoltaic arrays," NASA STI/Recon Technical Report N81, p. 11551,1979

[4] T. Townsend and L. Powers, "Photovoltaics and snow: An update from two winters of measurements in the sierra," in Proc. 37th IEEE Photovoltaic Specialists Conference, 2011, pp. 003231-003236.

[5] R. W. Andrews, A. Pollard, and J. M. Pearce, "The effects of snowfall on solar photovoltaic performance," Solar Energy, vol. 92, pp. 84-97, 2013.
[6] R. W. Andrews and J. M. Pearce, "Prediction of energy effects on photovoltaic systems due to snowfall events," in Proc. 38th IEEE Photovoltaic Specialists Conference, 2012, pp. 003386-003391.

[7] G. Wirth, M. Schroedter-Homscheidt, M. Zehner, and G. Becker, "Satellite-based snow identification and its impact on monitoring photovoltaic systems," Solar Energy, vol. 84, no. 2, pp. 215-226, 2010.

[8] Arduino playground. [Online]. Available: http://playground.arduino.cc/Code/TwitterLibrary

[9] Arduino tweet. [Online]. Available: http://arduino-tweet.appspot.com/

[10] Arduino search results. [Online]. Available: http://www.instructables.com/howto/arduino

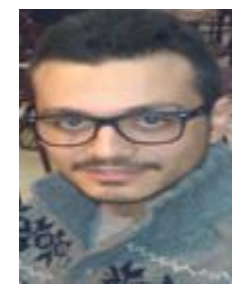

Seyedali Meghdadi was born in Tehran, Iran on September 19, 1987. He received the bachelor's degree of applied science in electrical engineeringpower in 2011. At present he is a master's student in renewable energy systems at Electrical Engineering Faculty, Memorial University of Newfoundland, NL, Canada.

Currently he is a research assistant at Memorial University of Newfoundland, NL, Canada. His current research interests are renewable energy systems, power systems and control strategies of hybrid energy systems.

M. Tariq Iqbal received the B.Sc. degree from the University of Engineering and Technology, Lahore in 1986, the M.Sc. degree in nuclear engineering from the Quaid-eAzam University, Islamabad in 1988 and the Ph.D. degree in electrical engineering from the Imperial College London in 1994. Since 2001 he is working at the Faculty of Engineering and Applied Science, Memorial University of Newfoundland. Presently he is a full professor. His teaching activities cover a range of electrical engineering topics. Currently, his research is focused on modeling and control of renewable energy systems with interests in the areas of design of control systems and comparison of control strategies of hybrid energy systems. 